\title{
Linear Oscillatory Cellular Thermocapillary Convection in Liquid Layers
}

\author{
Kai-Hua Guo* \\ Chinese Academy of Sciences, Guangzhou, People's Republic of China \\ and \\ Wen-Jei Yang $\dagger$ \\ University of Michigan, Ann Arbor, Michigan 48109
}

\begin{abstract}
A linear stability analysis is performed on a thermal, stratified liquid layer with a deformable thermocapillary surface. The objective is to investigate the possibility and conditions for existence of oscillatory cellular convection in the linear thermocapillary system. In general, the principle of the exchange of stabilities for the onset of cellular convection in liquid layers with deformable surfaces may not hold, especially for systems with small Prandtl numbers. This study discloses the characteristics of a linear oscillatory convection and associated interfacial wave in a thermocapillary liquid layer. The Marangoni number for the onset of oscillatory convection as overstability as well as the corresponding wave number and frequency for various Prandtl and Crispation numbers are determined based on the linear stability theory. The characteristics for overstability in the thermocapillary system are treated for both surface cooling and heating cases. The regions for existence of overstability and the effects of the Prandt and Crispation numbers are investigated.
\end{abstract}

\section{Nomenclature}

\footnotetext{
= arbitrary constant in Eqs. (14-16)

$=$ wave number; $a_{c}$, critical value

$=$ limitation wave number for overstability

$=$ arbitrary constant in Eqs. (14-16)

$=$ Crispation number, $=\nu \alpha / \ell \sigma$

= imaginary part of a complex number

$=$ thermal conductivity, $\mathrm{Wm}^{-10} \mathrm{C}^{-1}$

$=$ thickness of liquid layers, $\mathrm{m}$

$=$ Marangoni number, $=S(q / k) \ell^{2} / \mu \alpha ; M a_{c}$, critical

$=$ minimum Marangoni number for oscillation

in liquid layers with positive heat flux

$=$ Prandtl number, $=v / \alpha$

$=$ dimensionless pressure

$=$ heat flux, $\mathrm{kWm}^{-2}$

$=$ Rayleigh number

$=$ real part of a complex number

$=$ thermocapillarity, $=\partial \sigma / \partial T, \mathrm{Nm}^{-10} \mathrm{C}^{-1}$

$=$ temperature ${ }^{\circ} \mathrm{C} ; \bar{T}$, mean value

$=$ time, $\mathrm{s}$

$=x$ component of dimensionless velocity

$=$ dimensionless velocity vector

$=y$ component of dimensionless velocity

$=z$ component of dimensionless velocity

$=$ velocity amplitude of normal modes of disturbances

$=$ thermal diffusivity, $\mathrm{m}^{2} \mathrm{~s}^{-1}$

$=$ dimensionless surface deformation

$=$ amplitude of surface deformation

$=$ dimensionless temperature

$=$ temperature amplitude of normal modes of disturbances

$=$ dynamic viscosity, $\mathrm{Nsm}^{-2}$

$=$ kinematic viscosity, $\mathrm{m}^{2} \mathrm{~s}^{-1}$

$=$ surface tension, $\mathrm{Nm}^{-1}$

$=$ normal modes of disturbances defined by Eq. (13)
}

Received May 8, 1989; revision Nov. 21, 1989. Copyright (C) 1989 by the American Institute of Aeronautics and Astronautics, Inc. All rights reserved.

* Researcher, Institute of Energy Conversion.

$\uparrow$ Professor, Department of Mechanical Engineering and Applied Mechanics.
$\Omega \quad=$ complex growth rate of disturbances

$\omega=$ frequency of oscillation

$$
\begin{array}{ll}
\text { Subscripts } \\
c \quad=\text { critical } \\
\begin{array}{ll}
\operatorname{Im} & =\text { imaginary part } \\
\operatorname{Re} & =\text { real part } \\
x & =x \text { coordinate } \\
y & =y \text { coordinate }
\end{array}
\end{array}
$$

\section{Introduction}

$\mathbf{T}$ E classical problem of cellular convection in a fluid layer heated from below or cooled from above has been understood as a hydrodynamic instability phenomenon induced by unstable thermal stratification. This cellular convection phenomenon was first investigated by Benard ${ }^{1}$ and the flow pattern has commonly been referred to as Benard cells. After Rayleigh's ${ }^{2}$ linear stability analysis on a fluid layer with a negative temperature gradient, the phenomenon of Benard cells was understood due to the buoyancy-driving mechanism. Later, Block ${ }^{3}$ and Pearson ${ }^{4}$ found that the cellular convection observed by Benard ${ }^{1}$ was actually induced by the surface-tension gradient along the free surface. The gradient is induced by temperature disturbances excited under unstable thermal stratification, and it drives the liquid into convection through fluid shear stress. Recently, the Marangoni convection, including both thermocapillary and solute-capillary effects, as well as its associated interfacial phenomena have attracted considerable attention because of important applications in material processing and heat and mass transport in vapor-liquid or liquid-liquid interfacial regions where surface-tension effects are significant. ${ }^{\text {s-9 }}$

The onset of cellular thermocapillary convection in a fluid layer is governed by an instability criterion for the Marangoni number $M a$. Based on linear theory, infinitesimal disturbances in the system will grow exponentially when the Marangoni number exceeds its critial value and will decay otherwise. The instability criterion is then determined at the zero growth rate of the disturbances. In linear theory, the principle of the exchange of stabilities often plays an important role. This principle is valid for the Rayleigh-Benard problem (buoyancy-driven cellular convection) ${ }^{10}$ and the Marangoni instaity problem studied by Pearson ${ }^{4}$ and Vidal and Acrivos. ${ }^{11}$ In 
both studies, the free surface or vapor-liquid interface considered was flat and nondeformable. In reality, however, the interface can be deformed under the effects of normal and shear stresses unless it has an infinitely strong surface tension. Although its magnitude may be very small, the interfacial deformation can have a drastic effect on cellular thermocapillary convection, especially for thin liquid layers. This effect is often called the Crispation effect, which is characterized by the Crispation number $(\mathrm{Cr})$. Scriven and Sternling ${ }^{12}$ and Smith $^{13}$ investigated the Crispation effect and found that this effect can cause absolute instability at a small wave number in the absence of gravity. However, in their analyses, as well as many others after them, the principle of the exchange of stabilities was assumed valid without proof, and the possibility for existence of oscillatory convection and interfacial wave motion was totally ignored.

If the surface of a liquid layer is deformable, the principle of the exchange of stabilities will not be valid any more because the balance of normal stress together with the kinematic condition at the surface form a dynamic equation, which is wave-type in nature. Take an extreme situation for example. If the fluid viscosity is negligible $(\operatorname{Pr} \rightarrow 0)$, the flow can be treated as inviscid, and the interfacial dynamics will lead to gravity and capillary waves on the free surface. ${ }^{14}$ For a viscous fluid (with a finite Prandtl number), the viscous effect may damp out oscillation if there is no other driving force. However, in a thermocapillary system, the thermocapillarity will convert thermal energy into mechanical energy to accelerate oscillation and overcome viscous damping so that oscillation of both convection and interfacial wave may be maintained. Recently, Verlarde et al. ${ }^{15}$ studied interfacial oscillations in a semi-infinite liquid and found that thermocapillary effects can overtake viscous damping to sustain an oscillatory wave. However, they provide the overstability curves for only one small Prandtl number $(P r=0.1)$. The region for existence of oscillatory convection still remains undetermined. Takashima ${ }^{16,17}$ studied the effects of a free-surface deformation on the onset of surface-tension-driven instability in a horizontal thin liquid layer subject to a vertical temperature gradient using the linear stability theory. Both stationary convection ${ }^{16}$ and overstability ${ }^{17}$ were treated. It was concluded that 1) in the stationary convection case, the free-surface deformation is important only for unusually thin layers of very viscous liquids, and 2) in the overstability case, oscillatory modes of instability can occur when and only when the temperature of the solid wall is lower than that of the air phase.

While Takashima ${ }^{16,17}$ employed the Nield's model (both buoyancy and surface-tension mechanisms) ${ }^{18}$ to investigate overstability, the present work adopts the Pearson's model (surface-tension mechanism). ${ }^{4}$ The effects of the Prandtl and Crispation numbers on the onset of oscillatory convection and the conditions for existence of overstability in the thermocapillary system are determined in a broad range of $P r$ and $\mathrm{Cr}$ by means of linear stability analysis.

\section{Linear Analysis}

Consider a liquid of thickness $\ell$ unbounded in the $x-y$ plane. The $z$ coordinate points up with its origin at the base of the layer, which is a flat isothermal solid substrate. The top surface of the liquid layer is a deformable vapor-liquid interface. A linear thermal stratification (base state) is maintained by steady heat transfer through the liquid layer. There are two possibilities: the first is that the heat is transferred from the bottom to the top, i.e., the liquid layer is either heated from below or cooled from above (including evaporative cooling). Under this circumstance, the heat flux is positive, and, consequently, one has a positive Marangoni number $\mathrm{Ma}$. The second is that the heat is transferred from the top to the bottom, i.e., the layer is heated from the vapor side. In this case, the heat flux is negative and so is the Marangoni number (if the thermocapillarity $-\partial \sigma / \partial T$ is positive). Both circumstances are considered because the onset of oscillation as overstability is possible for both positive and negative Marangoni numbers. ${ }^{15}$ For the present purpose, the buoyancy effect is not taken into account, i.e., we assume the microgravity condition or when buoyancy forces are negligible compared to surface-tension forces $(R a / M a \ll 1)$. We also ignore the local surface cooling effect, which is caused by the disturbed surface heat flux due to surface-temperature disturbance. This disturbed heat flux tends to suppress the temperature disturbance on the surface and stabilizes the system. This stabilizing effect (characterized by the Biot number) on oscillatory convection will be similar to that on nonoscillatory marginal stability, which was studied in detail by Pearson ${ }^{4}$ and Scriven and Sternling. ${ }^{12}$ Therefore, the localcooling effect is not taken into account, and the Biot number is assumed zero. The vapor-liquid interface has a finite surface tension $\sigma$ which is temperature dependent such that the thermocapillarity of the interface is defined as $S=-\partial \sigma / \partial T$. The interface will have a wavy deformation under the effects of normal and shear stresses and surface tension. However, stresses from the vapor phase are negligible compared to those from the liquid phase; therefore they are assumed zero. The Boussinesq approximation is applicable here, i.e., all fluid properties are treated as constant, and the viscous dissipation in the energy equation is negligible.

Based on the above consideration, the linear Boussinesq equations (governing equations), in dimensionless form, read

$$
\begin{aligned}
& \nabla \cdot V=0 \\
& \frac{1}{P r} \frac{\partial \boldsymbol{V}}{\partial t}-\nabla^{2} \boldsymbol{V}=-\nabla p \\
& \frac{\partial \theta}{\partial t}-\nabla^{2} \theta=-\frac{\mathrm{d} \bar{T}}{\mathrm{~d} z} w
\end{aligned}
$$

where $\boldsymbol{V}=(u, v, w)$ is the velocity vector; $p$ and $\theta$ are disturbed pressure and temperature, respectively; $T$ is basic temperature, which is linearly disturbed in the $z$-direction due to steady heat transfer; and $\mathrm{d} \bar{T} / \mathrm{d} z=-1 ; \operatorname{Pr} \equiv \nu / \alpha$ is the Prandtl number where $v$ is the kinematic viscosity and $\alpha$ is the thermal diffusivity. The scale factor used for the nondimensionalization are $\ell$ for length, $\alpha / \ell$ for velocity, $\ell^{2} / \alpha$ for time, $\alpha \mu / \ell^{2}$ for pressure, and $q \ell / k$ for temperature.

The linear boundary conditions at the deformable thermocapillary interface $(z=1)$ are derived from momentum and energy balances. The force balances in the normal and tangential directions read, respectively,

$$
-p+2 \frac{\partial w}{\partial z}=\frac{1}{C r} \nabla_{\operatorname{Im}}^{2} \eta
$$

and

$$
-\frac{\partial^{2} w}{\partial z^{2}}+\nabla_{\mathrm{Im}}^{2} w=-M a \nabla_{\mathrm{Im}}^{2}(\theta-\eta)
$$

The energy condition is

$$
\frac{\partial \theta}{\partial z}=0
$$

and the kinematic condition at the wavy interface is

$$
\frac{\partial \eta}{\partial t}=w
$$

where $\nabla_{\operatorname{Im}} \equiv \boldsymbol{i}(\partial / \partial x)+\boldsymbol{j}(\partial / \partial y)$, and $\eta$ is the interfacial deformation variable, which is defined such that $z=1+\eta(x, y)$ at the thermocapillary interface. $M a \equiv S(q / k) \ell^{2} / \mu \alpha$ is the Marangoni number where $S$ is the thermocapillarity; $q$, heat 
flux; $k$, conductivity; $\mu$, dynamic viscosity; and $\ell$, layer thickness. $C r \equiv \mu \alpha / \ell \sigma$ is the Crispation number, which results from the force balance in the normal direction of the deformable interface.

On the solid substrate (at $z=0$ ), the no-slip, nonpenetrating, and isothermal conditions yield

$$
u=v=w=\frac{\partial w}{\partial z}=\theta=0
$$

The pressure term in the momentum equation [Eq. (2)] can be eliminated by multiplying the $x, y$, and $z$ components by $-\partial^{2} /(\partial x \partial z),-\partial^{2} /(\partial y \partial z)$, and $\nabla_{\operatorname{lm}}^{2}$, respectively, and adding them together. Upon substituting the flow continuity of Eq. (1), the resulting equation is

$$
\left(\frac{1}{\operatorname{Pr}} \frac{\partial}{\partial t}-\nabla^{2}\right) \nabla^{2} w=0
$$

The pressure in Eq. (4) can be expressed as

$$
\nabla_{\operatorname{Im}}^{2} p=\left(\frac{1}{\operatorname{Pr}} \frac{\partial}{\partial t}-\nabla^{2}\right) \frac{\partial w}{\partial z}
$$

Substituting Eq. (10) into Eq. (4), the normal force balance at $z=1$ becomes

$$
-\left(\frac{1}{\operatorname{Pr}} \frac{\partial}{\partial t}-3 \nabla^{2}\right) \frac{\partial w}{\partial z}-2 \frac{\partial^{3} w}{\partial z^{3}}=\frac{1}{C r} \nabla_{\operatorname{Im}}^{2}\left(\nabla_{\operatorname{Im}}^{2} \eta\right)
$$

Considering standing oscillatory waves, the normal mode solutions of the linear thermocapillary system take the form

$$
\begin{gathered}
w=\tilde{w}(z, t) \phi(x, y) \\
\theta=\tilde{\theta}(z, t) \phi(x, y) \\
\eta=\tilde{\eta}(t) \phi(x, y)
\end{gathered}
$$

$\phi(x, y)$ satisfies the relation ${ }^{10}$

$$
\nabla_{\operatorname{Im}}^{2} \phi(x, y)+a^{2} \phi(x, y)=0
$$

The general solution for $\phi$ takes the form of $\phi=e^{i\left(a_{x} x+a_{y} y\right)}$ with $a_{x}^{2}+a_{y}^{2}=a^{2}$. For the special case of a two-dimensional wave propagating in the $x$-direction, $a_{y}=0, a_{x}=a$, and $\phi=e^{i a x}$

From Eqs. (9) and (3), together with Eqs. (6-8), the solutions for $\tilde{w}, \tilde{\theta}$, and $\tilde{\eta}$ are found as

$$
\begin{aligned}
\tilde{w}= & {\left[A\left(\frac{a}{m} \sinh m z-\sinh a z\right)+B(\cosh m z-\cosh a z)\right] e^{\Omega t} } \\
\tilde{\theta}= & {[A(14)} \\
& +B\left(N \sinh r z-\frac{a \sinh m z}{m\left(m^{2}-r^{2}\right)}+\frac{\sinh a z}{a^{2}-r^{2}}\right) \\
&
\end{aligned}
$$

$$
\tilde{\eta}=\frac{1}{\Omega}\left[A\left(\frac{a}{m} \sinh m-\sinh a\right)+B(\cosh m-\cosh a)\right] e^{\Omega t}
$$

with

$$
\begin{gathered}
M=\frac{a}{r \cosh r}\left(\frac{\cosh m}{m^{2}-r^{2}}-\frac{\cosh a}{a^{2}-r^{2}}\right) \\
N=\frac{1}{r \cosh r}\left(\frac{m \sinh m-r \sinh r}{m^{2}-r^{2}}-\frac{a \sinh a-r \sinh r}{a^{2}-r^{2}}\right)
\end{gathered}
$$

where $m^{2}=a^{2}+\Omega / P r$ and $r^{2}=a^{2}+\Omega$. The $\Omega$ is a complex number, which governs the transient behavior of the distur- bance modes. The stability of the system is characterized by the real part of $\Omega[\operatorname{Re}(\Omega)]$. When $\operatorname{Re}(\Omega)>0$, the modes of disturbance will grow exponentially, and the system is unstable; otherwise it is stable. The condition for usual nonoscillatory marginal stability is determined at both $\operatorname{Re}(\Omega)=0$ and $\operatorname{Im}(\Omega)=0$ (the principle of the exchange of stability). If $\operatorname{Re}(\Omega)=0$ while $\operatorname{Im}(\Omega)$ is not equal to zero, the disturbances will oscillate in a bounded "circle" with a frequency $\omega=\operatorname{Im}(\Omega)$. Then the onset of oscillatory convection is called overstability. For the onset of convection as overstability, $\Omega=i \omega$, and then $m^{2}=a^{2}+i(\omega / P r)$ and $r^{2}=a^{2}+i \omega$. Physically, both $a$ (wave number) and $\omega$ (frequency) are required to be positive real numbers.

Boundary conditions of Eqs. (5) and (11) are now used to determine the arbitrary constants $A$ and $B$ and to yield a homogeneous linear system of equation. The condition for existence of non-trivial solutions leads to the characteristic equation for overstability of the linear system:

$$
\begin{aligned}
& \operatorname{Cr}\left(G_{1} R_{2}-G_{2} R_{1}\right)-i \frac{a}{\omega}\left(Q_{1} R_{2}-Q_{2} R_{1}\right) \\
& \quad+\operatorname{Ma}\left[\operatorname{Cr}\left(G_{1} F_{2}-G_{2} F_{1}\right)-i \frac{a}{\omega}\left(Q_{1} F_{2}-Q_{2} F_{1}\right)\right]=0
\end{aligned}
$$

where

$$
\begin{aligned}
& Q_{1}=\frac{a}{m} \sinh m-\sinh a \\
& Q_{2}=\cosh m-\cosh a \\
& R_{1}=2 Q_{1}-i \frac{\omega}{a^{2} P r} \\
& R_{2}=2 Q_{2}-i \frac{\omega}{a^{2} P r} \\
& G_{1}=2 Q_{2}-i \frac{\omega \cosh a}{a^{2} P r} \\
& G_{2}=2\left(\frac{m}{a} \sinh m-\sinh a\right)-i \frac{\omega \sinh a}{a^{2} \operatorname{Pr}} \\
& F_{1}=M \sinh r+i \frac{\operatorname{Pr}}{\omega}\left(\frac{a \sinh m}{m(1-P r)}+\frac{\sinh a}{\operatorname{Pr}}+\frac{Q_{1}}{\operatorname{Pr}}\right) \\
& F_{2}=N \sinh r+i \frac{\operatorname{Pr}}{\omega}\left(\frac{\cosh m-\cosh r}{1-\operatorname{Pr}}+\frac{\cosh a-\cosh r}{\operatorname{Pr}}+\frac{Q_{2}}{\operatorname{Pr}}\right)
\end{aligned}
$$

\section{Results and Discussion}

Equation (17) characterizes the stability of the thermocapillary system and the behaviors of the thermocapillary or capillary wave in the finite liquid layer. In the absence of thermocapillarity, or for an isothermal liquid $(M a=0)$, the equation reduces to

$$
\omega^{2}=\frac{a^{3} P r}{C r} \tanh a
$$

for negligible fluid viscosity. This is the dimensionless dispersion relation for capillary waves in an inviscid liquid layer with a finite depth. ${ }^{14}$

For the general circumstances where $M a$ and $P r$ are nonzero, Eq. (17) provides the characteristics for overstability of the linear system. In the limit of vanishing $w$, this relation can be reduced to

$$
M a=\frac{8 a^{2} \cosh a(\cosh a \sinh a-a)}{\sinh ^{3} a-a^{3} \cosh a+8 C r a^{3} \cosh a}
$$

which is the characteristic equation for nonoscillatory marginal stability as obtained by Scriven and Sternling. ${ }^{12}$ 
With $w$ being real and positive, Eq. (17) can be rewritten in the explicit form of $M a$ (eigenvalue) as

$$
M a=2 \omega^{2} \frac{\left(J_{1 \mathrm{Re}}+i J_{1 \mathrm{Im}}\right)+\frac{2 C r}{a}\left(J_{2 \mathrm{Re}}+i J_{2 \mathrm{Im}}\right)}{\left(K_{1 \mathrm{Re}}+i K_{1 \mathrm{Im}}\right)+\frac{2 C r}{a}\left(K_{2 \mathrm{Re}}+i K_{2 \mathrm{Im}}\right)}
$$

where $J$ and $K$ are real functions of $a, w$, and $P r$. Since the Marangoni number is real, the imaginary part of the right side of Eq. (20) must be zero. Then one has

$$
\begin{aligned}
& \left(J_{1 \mathrm{Re}}+\frac{2 C r}{a} J_{2 \mathrm{Re}}\right)\left(K_{1 \mathrm{Im}}+\frac{2 C r}{a} K_{2 \mathrm{Im}}\right) \\
& -\left(J_{1 \mathrm{Im}}+\frac{2 C r}{a} J_{2 \mathrm{Im}}\right)\left(K_{1 \mathrm{Re}}+\frac{2 C r}{a} K_{2 \mathrm{Re}}\right)=0 \\
& M a=2 \omega^{2} \frac{\left(J_{\mathrm{IRe}}+\frac{2 C r}{a} J_{2 \mathrm{Re}}\right)\left(K_{\mathrm{IRe}}+\frac{2 C r}{a} K_{2 \mathrm{Re}}\right)+\left(J_{1 \mathrm{Im}}+\frac{2 C r}{a} J_{2 \mathrm{Im}}\right)\left(K_{1 \mathrm{Im}}+\frac{2 C r}{a} K_{2 \mathrm{Im}}\right)}{\left(K_{1 \mathrm{Re}}+\frac{2 C r}{a} K_{2 \mathrm{Re}}\right)^{2}+\left(K_{1 \mathrm{Im}}+\frac{2 C r}{a} K_{2 \mathrm{Im}}\right)^{2}}
\end{aligned}
$$

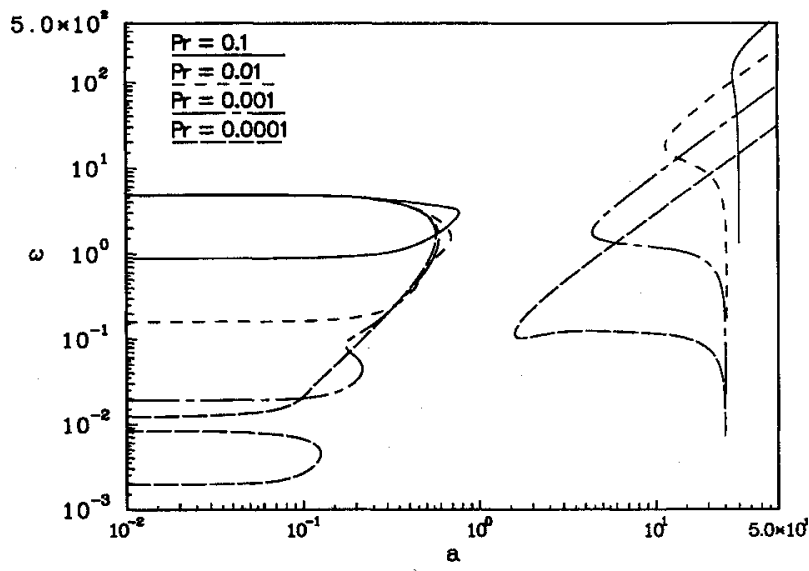

Fig. 1 Overstability loci $\omega$ vs $a$ for liquid layers with $C r=0.01$ and
Equation (21) provides the condition for existence of neutral oscillatory modes. With $\mathrm{Pr}, \mathrm{Cr}$, and $a$ given, if the left side of Eq. (21) has a nonzero root of $w$, the oscillatory mode will exist with frequency $\omega$. Otherwise, oscillation is impossible, i.e., $\operatorname{Im}(\Omega)$ must be zero when $\operatorname{Re}(\Omega)=0$ (the principle of the exchange of stabilities). In the special case of a liquid layer with a nondeformable surface, i.e., $C r=0$, no nonzero root of $w$ can be found from Eq. (21). The conclusion by Vidal and Acrivos ${ }^{11}$ is thus confirmed.

In determination of the characteristics for overstability, Eq. (21) is used to determine the frequency $\omega$ for the existent oscillatory mode as a function of wave number $a$ with $\mathrm{Cr}$ and $P x$ as parameters. This provides the overstability loci on the $\omega-a$ plane. The eigenvalue $M a$ is then calculated by using Eq. (22). This yields the overstability curves for $M a$ as a function of $a$. Figures 1 and 2 show the results for various $\mathrm{Pr}$ at $C r=0.01$. In Fig. 1, the overstability locus for each Prandt number has two separate branches: the upper branch lies in the larger wave number and relatively higher frequency region; and the lower branch is in the smaller wave number and relatively lower frequency region. The upper branches have positive eigenvalues $(M a)$, as shown in Fig. 2a where the lower part of the curve corresponds to the upper part (higher $\omega)$ of the upper branch at the same $\operatorname{Pr}$ in Fig. 1, whereas the lower branches have negative $M a$, as shown in Fig. $2 \mathrm{~b}$ where the upper part of the curve corresponds to the upper part (higher $\omega$ ) of the corresponding lower branch. There is a minimum wave number for each upper branch and a maximum wave number for each lower branch. These extreme wave numbers are called the limitation wave numbers $a_{\mathrm{lim}}$. Oscillation is impossible for $a<a_{\text {lim }}$ if the Marangoni number is positive (a liquid layer with positive heat flux and positive thermocapillarity $S$ ) and impossible for $a>a_{\lim }$ if the Marangoni number is negative (a liquid layer with negative heat flux and positive thermocapillarity $S$ ). It can be seen that each overstability curve in Fig. 2a merges into the marginal stability curve for steady modes (the heavy solid curve) at a wave number at which the frequency drops to zero, i.e., the characteristic equation for overstability will be reduced to that for nonoscillatory instability as $\omega \rightarrow 0$. In Fig. 2a, the minimum Marangoni number for each overstability curve is not considered as a critical Marangoni number because the nonoscillatory modes may become unstable below this value, and it is denoted as $M a_{\omega}$. When $M a<M a_{\omega}$, no oscillation is possible in the liquid layer with positive heat flux and positive thermocapillarity.
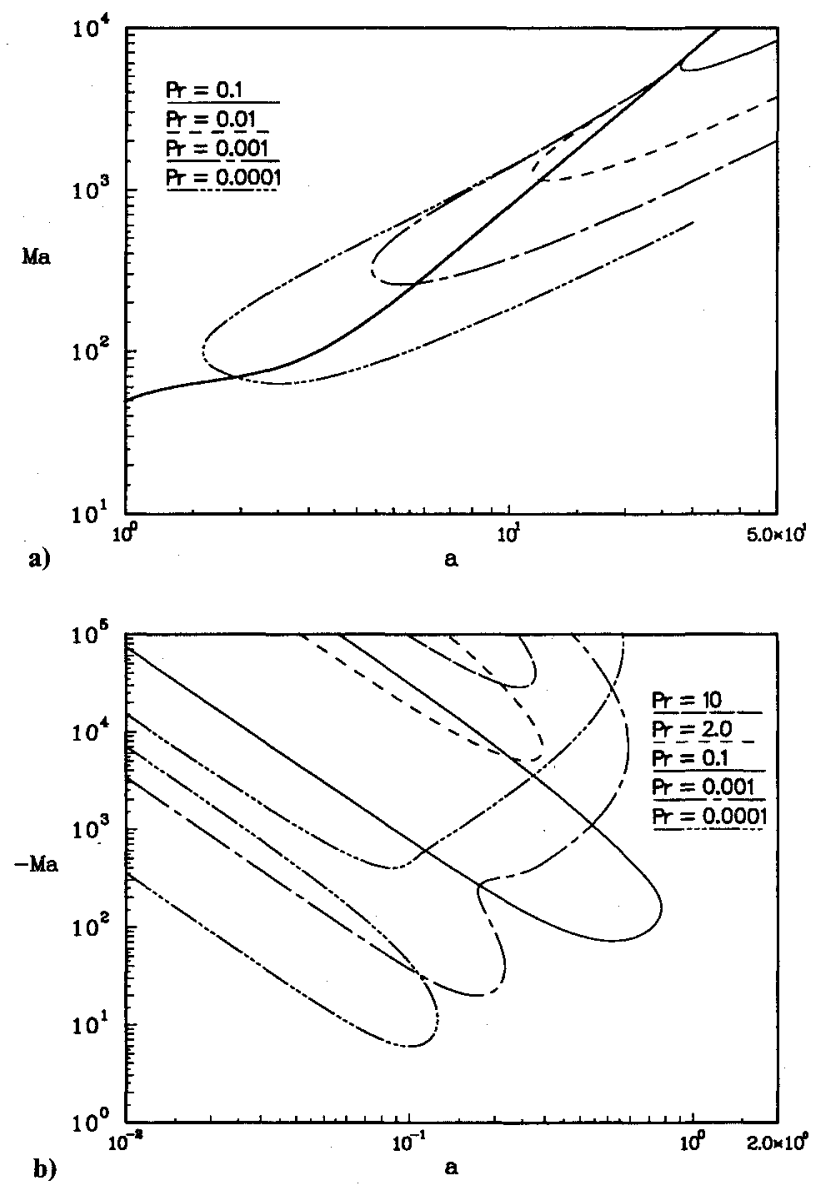

Fig. 2 Overstability characteristic curves $M a$ vs $a$ for liquid layers with $\mathrm{Cr}=0.01$ and various $\mathrm{Pr}$ : a) positive eigenvalue $(\mathrm{Ma}>0)$, the heavy solid line is the steady marginal stability curve; b) negative eigenvalue $(M a<0)$

In the case with a negative eigenvalue $M a$ (a liquid layer subjected to a negative heat flux and with a positive thermocapillarity $S$ ), oscillatory convection and interfacial wave can be sustained when the absolute value of the Marangoni number exceeds a critical value $M a_{c}$ (the minimum point in each curve in Fig. 2b). It can be seen that the Prandtl number 


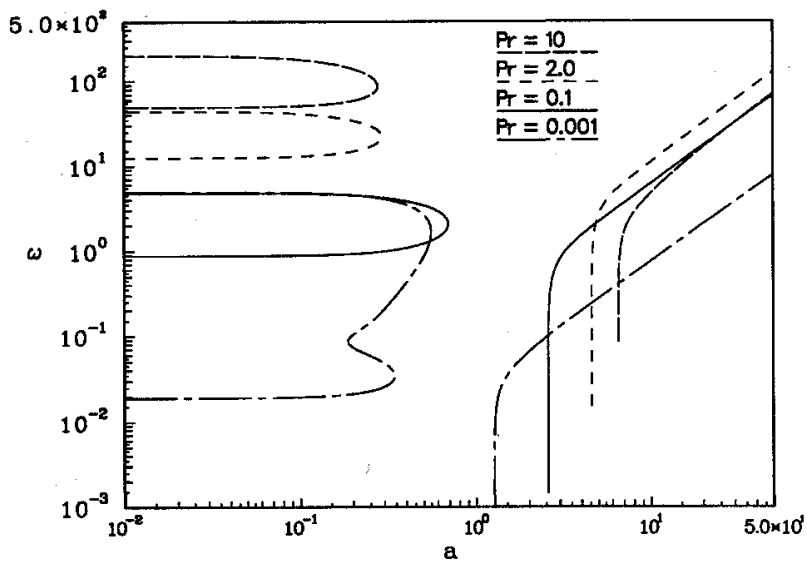

Fig. 3 Overstability loci $\omega$ vs $a$ for liquid layers with $C r=1$ and various $\mathrm{Pr}$.
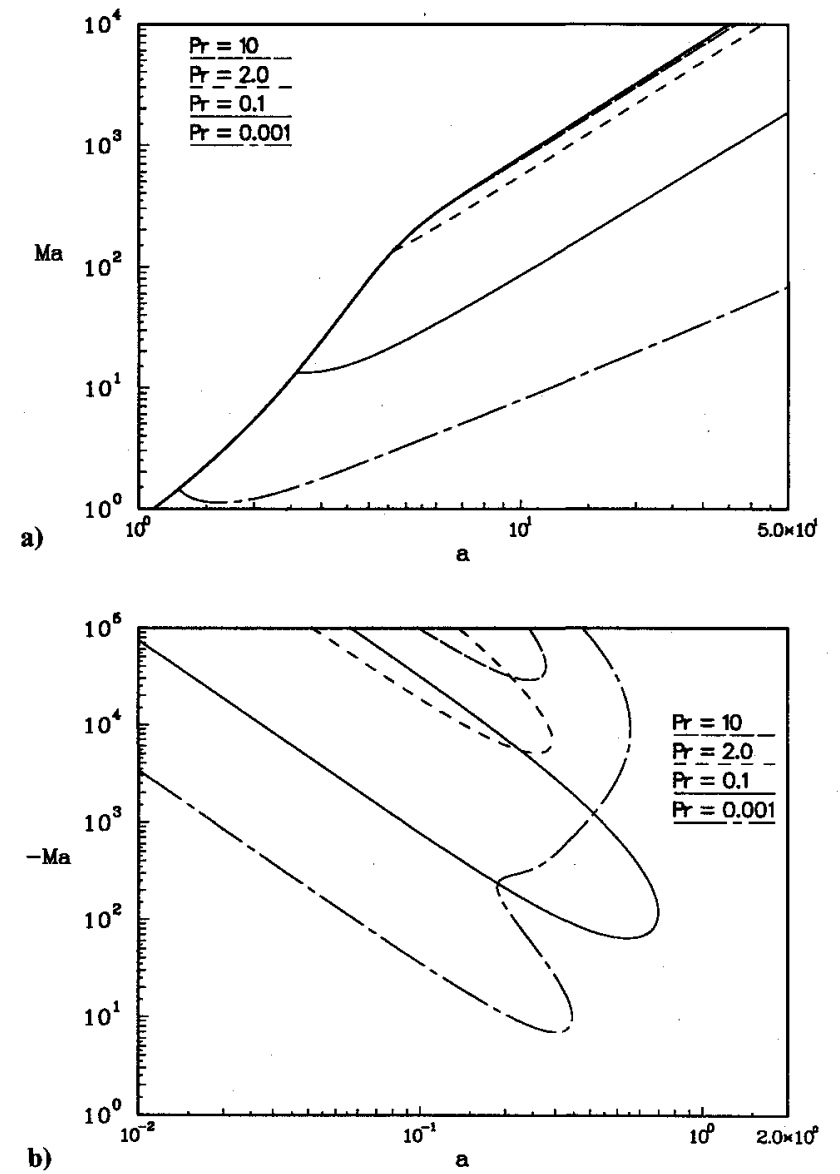

Fig. 4 Overstability characteristic curves $M a$ vs $a$ for liquid layers with $C r=1$ and various $P r$ : a) positive eigenvalue $(M a>0)$, the heavy solid line is the steady marginal stability curve; b) negative eigenvalue $(M a<0)$.

has a significant effect on the critical Marangoni number. When $\operatorname{Pr}>2 M a_{c}$ at the onset of oscillation as overstability is more than $10^{4}$. It is also noted that the lower branch starts to be separated when $P r$ becomes small $(P r=0.001)$. When $P r=0.0001$, the branch is separated into two as shown in Fig. 1 and Fig. 2b. In fact, there is one more branch for each $\mathrm{Pr}$ in the negative $\mathrm{Ma}$ case. Those branches only appear in extremely high $\mathrm{Ma}$ and frequency; therefore, they are not seen. By examination of Fig. 2, it can be seen that the oscillatory convection with a negative eigenvalue is more likely to occur than that with a positive eigenvalue. For example, at $P r=0.001$, the critical Marangoni number $M a_{c}$ is about 20 for the negative-heat-flux case, whereas the minimum Marangoni number $M a_{\omega}$ for sustaining oscillation in the positive-heat-flux case is about 200. At $\operatorname{Pr}=0.1, M a_{c}$ is less than 100, whereas $M a_{\omega}$ is more than 5000 .

The effects of the Crispation number on the characteristics for overstability are shown in Fig. 3-7. For a large Crispation number $(C r=1)$, as depicted in Figs. 3 and 4, both $M a_{c}$ and $M a_{\omega}$ are reduced significantly and the system is very easy to be disturbed and to become unstable. In the positive-heat-flux case, the overstability curves merge into the nonoscillatory stability curve at their limitation wave numbers. The curves for the negative-heat-flux case are similar to those for $C r=0.01$ except $M a_{c}$ becomes much smaller. Figures 5 and 6 show the characteristic curves for overstability at a small Crispation number $(\mathrm{Cr}=0.001)$. Oscillation is impossible for systems with positive $M$ unless the wave number is very large $(a \geq 50)$. For the negative-heat-flux case, the overstability curves with $\mathrm{Pr}<1$ are rather different. The loci on the $\omega-a$ plane rush in the region of $a>1$ (in Fig. 5) and the overstability curves (in Fig. 6) possess more than one minimum point. This peculiar behavior of the characteristics for overstability may be due to a strong interaction between the surface elasticity and thermocapillarity, i.e., the waves with larger wave numbers accelerated by thermocapillarity are now easier to match with the natural frequency of the capillary surface. For $C r<0.001$, oscillation in systems with negative $M a$ can occur over a wide range of $a$ and $\omega$ when $P r$ is small. Figure 7 shows the overstability curves for various small $\mathrm{Cr}$ at $\operatorname{Pr}=0.01$.

Figure 8 shows the region for existence of oscillation on the Pr-a plane, in which the curves indicate the variation of the limitation wave number with $P r$. The curves named $-M a$ are

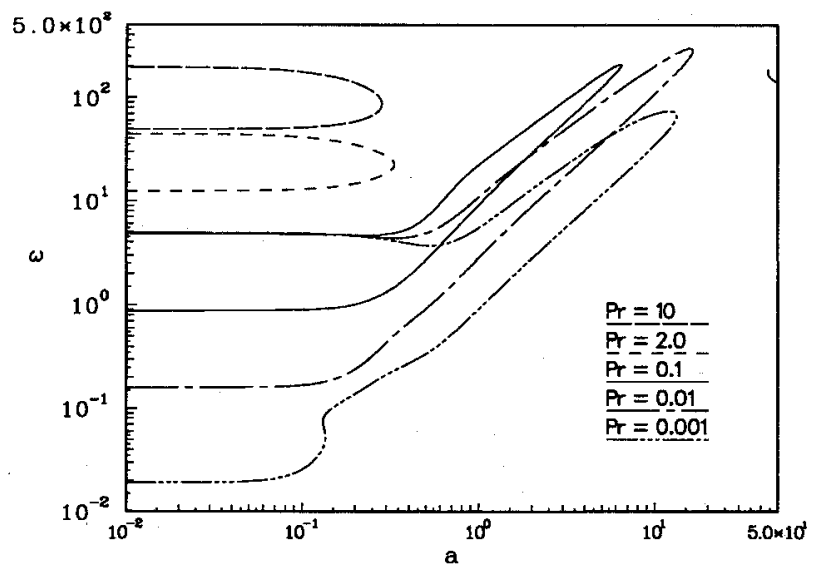

Fig. 5 Overstability loci $\omega$ vs $a$ for liquid layers with $C r=0.001$ and various $\mathrm{Pr}$.

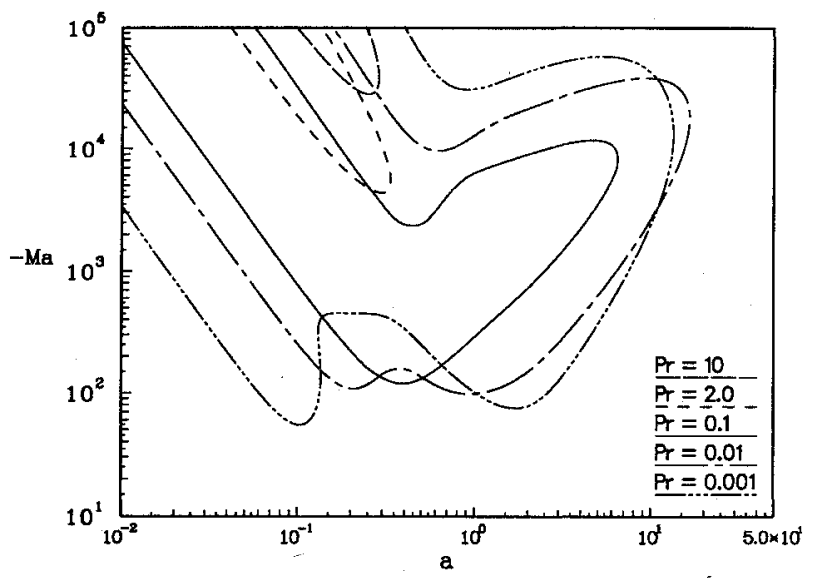

Fig. 6 Overstability characteristc curves $M a$ vs $a$ for liquid layers with $C r=0.001$ and various $\operatorname{Pr}$ (negative eigenvalue $M a<0$ ). 


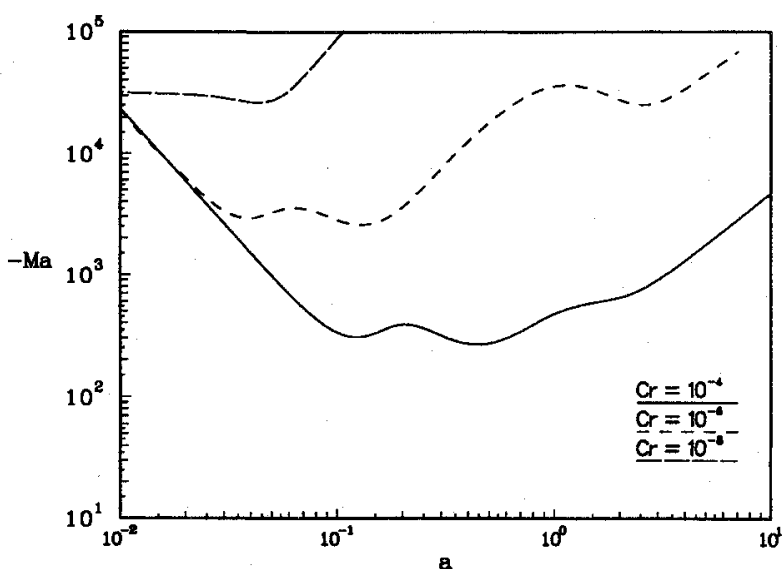

Fig. 7 Overstability characteristic curves with negative eigenvalue $-M a$ vs $a$ for small Crispation numbers with $P r=0.01$.

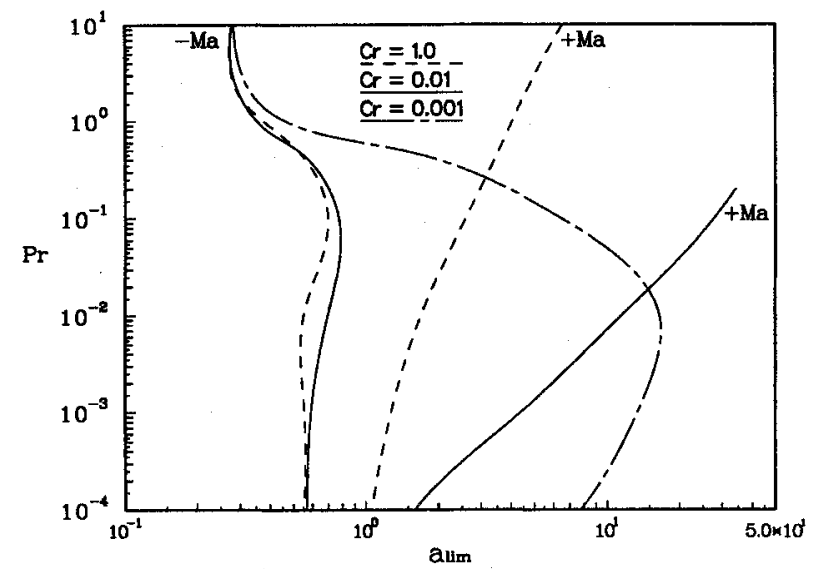

Fig. 8 Dependency of limitation wave number on Prandtl number for liquid layers with various $\mathrm{Cr}$.

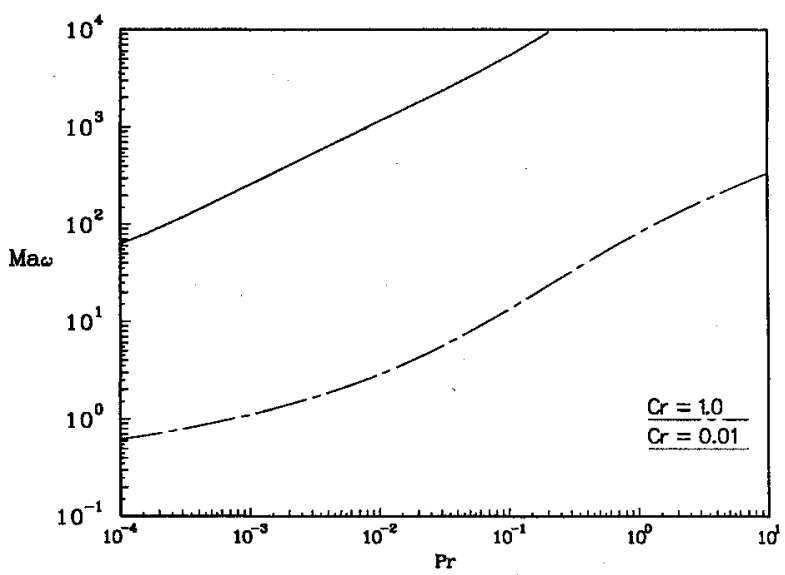

Fig. 9 Variation of minimum Marangoni number with Prandtl number for oscillation in liquid layers with positive $M a$.

for systems with negative $M a$. For those systems, no oscillation can exist in the region to the right of the $-M a$ curves. The $+M a$ curves are for systems with positive $M a$, and no oscillation can be sustained in the region to the left of them. For a liquid layer with positive $M a$ and $P r>1$, oscillation is almost impossible except for unreasonably large wave numbers and very high Marangoni numbers when $\mathrm{Cr}$ is less than 0.01 . For large $C r$, e.g., $C r=1$, overstability is possible for $a<10$ but confined in a very narrow region on the $M a-a$ diagram (see Fig. 4a). Therefore, it is unlikely to have oscillatory convection sustained in the system with $M a>0$ when $\operatorname{Pr}>1$, and the principle of the exchange of stabilities may be

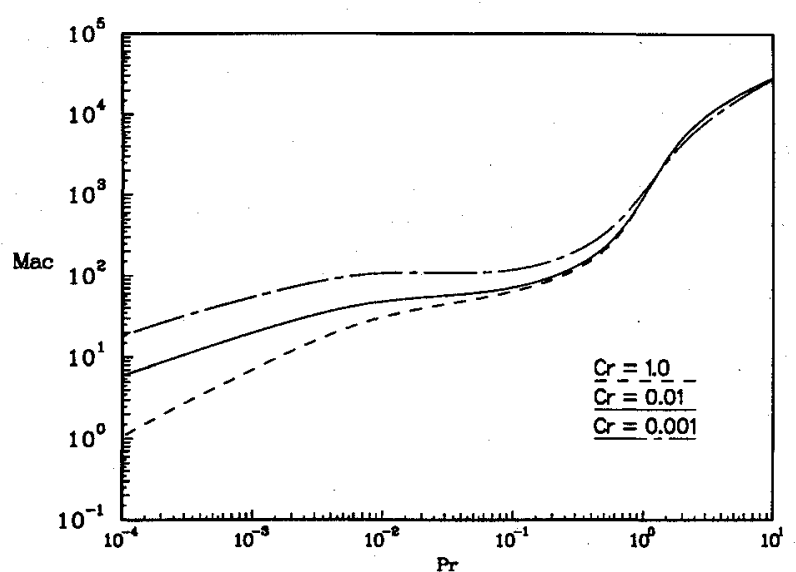

Fig. 10 Variation of critical Marangoni number with Prandtl number for oscillation in liquid layers with negative $\mathbf{M a}$.

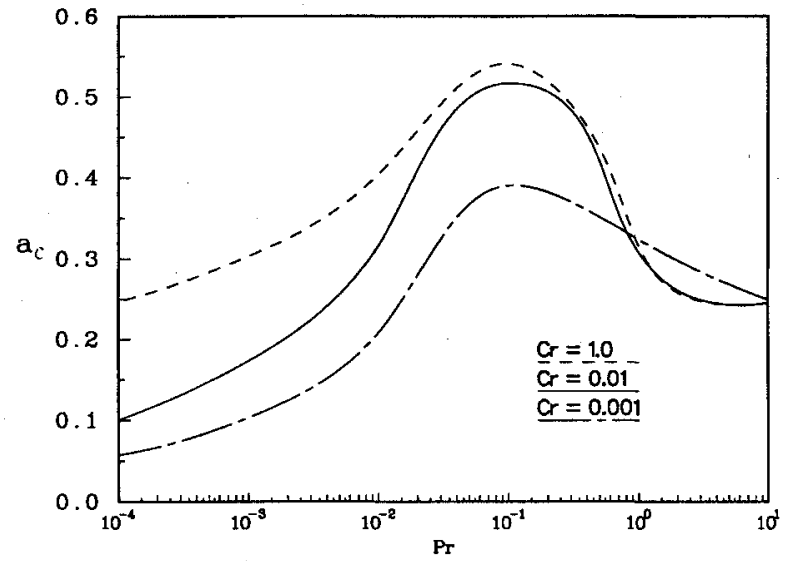

Fig. 11 Dependency of critical wave number on Prandtl number for liquid layers with negative $M a$.

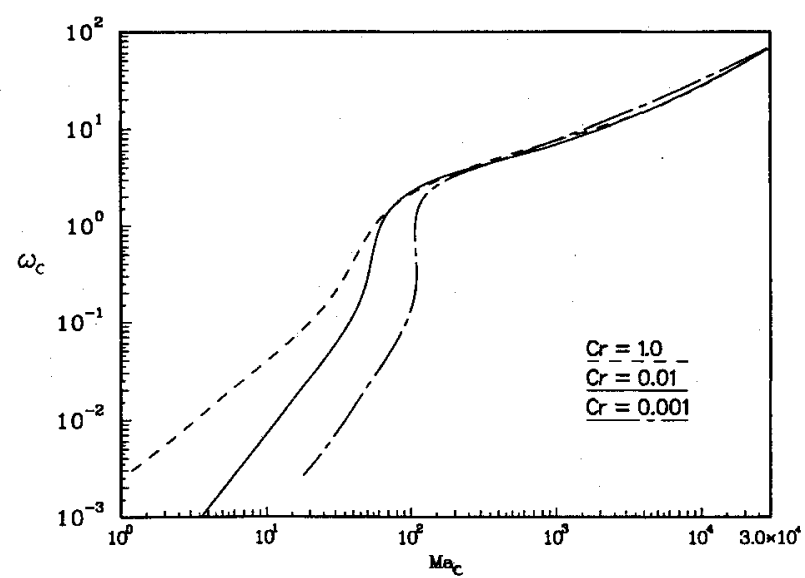

Fig. 12 Frequency of oscillation at critical state for liquid layers with negative $M a$.

assumed valid in analysis of such a system. For small $\mathrm{Pr}$, $\operatorname{Pr} \leq 0.1$, oscillation may occur in systems with positive $M a$ when $\mathrm{Cr} \geq 0.01$. The smaller the Prandtl number, the easier oscillation comes. The dependency of the minimum Marangoni number $M a_{\omega}$, on $P r$ is shown in Fig. 9. For layers with negative $M a$, however, oscillation can be sustained much easier, but the critical Marangoni number increases significantly when $P r$ is increased. When $P r>1, M a_{c}$ for the onset of oscillation is about $10^{3}$ or higher. The dependency of $M a_{c}$ on $P r$ is depicted in Fig. 10. The variation of the critical wave number $a_{c}$ with $P r$ is shown in Fig. 11. The largest $a_{c}$ appears around $\operatorname{Pr}=0.1$ where oscillation is most likely to occur. 
The frequency of oscillation at the critical point is shown in Fig. 12. The higher the frequency is, the larger the Marangoni number is needed to be since more energy is needed to sustain oscillation. It is observed that the frequency varies in proportion to $P$ and inversely to $\mathrm{Cr}$ as $\omega \sim \operatorname{Pr} / \mathrm{Cr}$. Therefore, the interfacial wave associated with the oscillatory convection in the thermocapillary system is a surface-tension wave in nature, whereas the thermocapillarity is merely to convert thermal energy into mechanica! energy, which provides the power needed for oscillation.

\section{References}

'Benard, H., "Les Tourbillons Cellulaires dans une Nappe Liquide Transportant de la Chaleur par Convection en Regime Permanent," Ann. Chim. Phys., Vol. 23, 1901, pp. 62-144.

${ }^{2}$ Rayleigh, L., "On Convection Currents in a Horizontal Layer of Fluid When the High Temperature is on the Under Side," Philosophical Magazine, Vol. 32, No. 192, 1916, pp. 529-546.

${ }^{3}$ Block, M. J., "Surface Tension as the Cause of Benard Cells and Surface Deformation in Liquid Film," Nature, Vol. 178, Sept. 22, 1956, pp. 650-651.

${ }^{4}$ Pearson, J. R. A., "On Convective Cells Induced by Surface Tension," Journal of Fluid Mechanics, Vol. 4, Sept. 1958, pp. 489 500 .

${ }^{5}$ Scharmann, A., Schuabe, D., Preisser, F., and Oeder, R., "Experiments on the Relevance of Marangoni Convection for Material Science in Space," (COSPAR) Space Research, Vol. 19, 1978, pp. $555-558$.

${ }^{6}$ Chun, C. H., and Wuest, W., "Experiments on the Transition from the Steady to the Oscillatory Marangoni-Convection of a Floating Zone under Reduced Gravity Effect," Acta Astronautica, Vol. 6, No. 9, 1979, pp. 1073-1082.

${ }^{7}$ Verlarde, M. G., and Castillo, J. L., "Transport and Reactive Phenomena Leading to Interfacial Instability," Convective Transport and Instability Phenomena, edited by J. Zierep, H. Oertel, G. Braun and Karlsruhe, 1982, pp. 235-246.

${ }^{8}$ Simon, B., and Panttaloni, J., "Convection Effects During the Crystal Growth of Sodium Chlorate from Aqueous Solutions," Physiocochemical Hydrodynamics, Vol. 5, No. 1, 1984, pp. 19-27.

${ }^{9}$ Uemura, T., and Yang, W. J., "Interfacial Turbulence in Evaporating Sessile Drops by a Combined Shadowgraph-Schlieren Method," Flow Visualization, edited by C. Veret, Hemisphere, Washington, DC, Vol. 4, 1987, pp. 79-84.

${ }^{10}$ Chandrasekhar, S., Hydrodynamic and Hydromagnetic Stability, Oxford University Press, London, 1961.

${ }^{11}$ Vidal, A., and Acrivos, A., "Nature of the Neutral State in Surface-Tension Driven Convection," Physics of Fluids, Vol. 9, No. 3, 1966, pp. 615-616.

${ }^{12}$ Scriven, L. E., and Sternling, C. V., "On Cellular Convection Driven by Surface-Tension Gradient: Effect of Mean Surface Tension and Surface Viscosity," Journal of Fluid Mechanics., Vol. 19, July 1964 , pp. 321-340.

${ }^{13}$ Smith, K. A., "On Convective Instability Induced by Surface Tension Gradients," Journal of Fluid Mechanics., Vol. 24, Feb. 1966 pp. $40 \mathrm{I}-414$.

${ }^{14}$ Yih, C. S., Fluid Mechanics, Westriver Press, Ann Arbor, MI, 1967.

${ }^{15}$ Velarde, M. G., Garcia, Y., and Castillo, J. L., "Interfacial Oscillations in Benard-Marangoni Layers," $\mathrm{PCH}$ Physico Chemical Hydrodynamics, Vol. 9, 1987, pp. 387-392.

${ }^{16}$ Takashima, M., "Surface Tension Driven Instability in a Horizontal Liquid Layer with a Deformable Free Surface. I. Stationary Convection," Journal of the Physical Society of Japan, Vol. 50, No. 8, 1981, pp. 2745-2750.

${ }^{17}$ Takashima, M., "Surface Tension Driven Instability in a Horizontal Liquid Layer with a Deformable Free Surface. II. Overstability," Journal of the Physical Society of Japan, Vol. 50, No. 8, 1981, pp. $2751-2756$

18 Nield, D. A., "Surface Tension and Buoyancy Effects in Cellular Convection," Journal of Fluid Mechanics, Vol. 19, 1964, pp. 341-352.

\title{
Dynamics of Reactive Systems, Part I: Flames and Part II: Heterogeneous Combustion and Applications and Dynamics of Explosions
}

\author{
A.L. Kuhl, J.R. Bowen, J.C. Leyer, A. Borisov, editors
}

Companion volumes, these books embrace the topics of explosions, detonations, shock phenomena, and reactive flow. In addition, they cover the gasdynamic aspect of nonsteady flow in combustion systems, the fluid-mechanical aspects of combustion (with particular emphasis on the effects of turbulence), and diagnostic techniques used to study combustion phenomena.

Dynamics of Explosions (V-114) primarily concerns the interrelationship between the rate processes of energy deposition in a compressible medium and the concurrent nonsteady flow as it typically occurs in explosion phenomena. Dynamics of Reactive Systems (V-113) spans a broader area, encompassing the processes coupling the dynamics of fluid flow and molecular transformations in reactive media, occurring in any combustion system.

To Order, Write, Phone, or FAX:

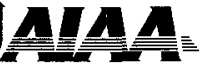

American Institute of Aeronautics and Astronautics c/o TASCO

9 Jay Gould Ct., P.O. Box 753, Waldorf, MD 20604

Phone (301) 645-5643 Dept. 415 FAX (301) 843-0159
V.113 1988865 pp., 2-vols. Hardback ISBN 0-930403-46-0

AIAA Members $\$ 92.95$

Nonmembers $\$ 135.00$
V.114 1988540 pp. Hardback ISBN 0-930403-47-9

AIAA Members $\$ 54.95$

Nonmembers $\$ 92.95$

Postage and Handling $\$ 4.75$ for $1-4$ books (call for rates for higher quantities). Sales tax: $\mathrm{CA}$ residents add $7 \%$, DC residents add $6 \%$. All orders under $\$ 50$ must be prepaid. All foreign orders must be prepaid. Please allow 4 weeks for delivery. Prices are subject to change without notice. 